Check for updates

London, UK

Cite this as: BMJ 2021;372:n326 http://dx.doi.org/10.1136/bmj.n326 Published: 03 February 2021

\section{Covid-19: New data on Oxford AstraZeneca vaccine backs 12 week dosing interval}

\author{
Jacqui Wise
}

The UK's approach of leaving an interval of three months between doses of the Oxford AstraZeneca covid-19 vaccine has been supported by new data, with the Oxford University researchers also saying the vaccine "may have a substantial impact on transmission."

The paper, a preprint currently under review at the Lancet, is an analysis of additional data from trials involving 17177 participants in the UK, Brazil, and South Africa. ${ }^{1}$ It includes the results of a further month of data collection with 332 cases of symptomatic covid-19-an additional 201 cases than were previously reported. ${ }^{2}$

A single standard dose of vaccine provided $76 \%$ protection overall against symptomatic covid-19 in the first 90 days after vaccination with protection not falling in this time frame. It is not clear, however, how long protection might last with a single dose as there were too few cases after 90 days to make any meaningful judgment.

The analyses suggest that it is the dosing interval and not the dosing level which has the greatest impact on the efficacy of the vaccine. This is in line with previous research supporting greater efficacy with longer intervals with other vaccines such as influenza and Ebola.

The study found vaccine efficacy reached $82.4 \%$ after a second dose in those with a dosing interval of 12 weeks or more ( $95 \%$ confidence interval $62.7 \%$ to $91.7 \%$ ). If the two doses were given less than six weeks apart the efficacy was only $54.9 \%$ (CI $32.7 \%$ to $69.7 \%)$.

As part of the UK arm of the trial, the researchers obtained weekly nose and throat swabs from volunteers to check for infections. The vaccine appears quite poor at preventing asymptomatic infection; its efficacy increased with a longer gap between doses but the confidence intervals were wide.

However, the researchers found that after a single dose of vaccine, overall polymerase chain reaction test positive cases of covid-19 (both symptomatic and asymptomatic) fell by $67 \%$, raising hopes that it may have a substantial impact on transmission by reducing the number of infected people in the population.

Andrew Pollard, chief investigator of the Oxford vaccine trial, and co-author of the paper, said that the new data "supports the policy recommendation made by the Joint Committee on Vaccination and Immunisation for a 12 week prime boost interval, as they look for the optimal approach to rollout, and reassures us that people are protected from 22 days after a single dose of the vaccine."

Commenting on the study, Paul Hunter, professor in medicine at the University of East Anglia, said, "Taking all this evidence together, the 12 week gap between first and second dose is clearly the better strategy as more people can be protected more quickly and the ultimate protective effect is greater. Given the poor efficacy at preventing asymptomatic infections, the vaccine will not stop transmission of covid but will still go a long way to reduce the $R$ value and transmission because there will be far fewer symptomatic infections and people who are symptomatic are rather more infectious than those who are asymptomatic."

Azra Ghani, chair in infectious disease epidemiology at Imperial College London, urged caution over the results, however, pointing out that the study was not designed to look at different dosing gaps or at one versus two doses. "Participants who received a single dose were younger, more likely to be female, more likely to be a healthcare worker, more likely to be resident in Brazil, and more likely to be white than those who received two doses. In addition, those who received a single dose were followed for a

significantly longer period of time," she said. "This means that it is not sensible to compare the efficacy estimates from a single dose with those from two doses.”

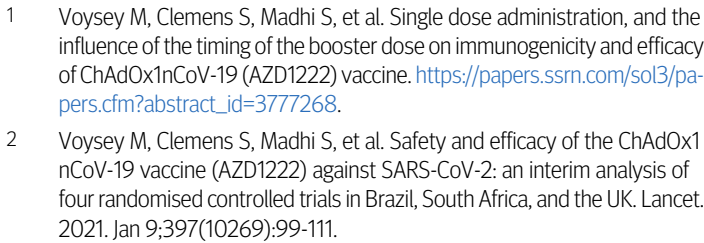

Voysey M, Clemens S, Madhi S, et al. Single dose administration, and the influence of the timing of the booster dose on immunogenicity and efficacy of ChAdOx1nCoV-19 (AZD1222) vaccine. https://papers.ssrn.com/sol3/papers.cfm?abstract_id=3777268.

2 Voysey M Clemens S, Madhi S, et al. Safety and efficacy of the ChAdOx $n C o V-19$ vaccine (AZD1222) against SARS-CoV-2: an interim analysis of four randomised controlled trials in Brazil, South Africa, and the UK. Lancet. 2021. Jan 9;397(10269):99-111.

This article is made freely available for use in accordance with BMJ's website terms and conditions for the duration of the covid-19 pandemic or until otherwise determined by BM]. You may use, download and print the article for any lawful, non-commercial purpose (including text and data mining) provided that all copyright notices and trade marks are retained. 